\title{
Review
}

Journal of Innate

Immunity
J Innate Immun 2013;5:427-433

DOI: $10.1159 / 000351979$
Received: September 1, 2012

Accepted after revision: May 14, 2013

Published online: June 11, 2013

\section{The Mechanism and Physiological Function of Macroautophagy}

\author{
Daniel J. Klionsky ${ }^{a-c}$ Patrice Codogno ${ }^{d}$ \\ a Life Sciences Institute, and Departments of ${ }^{\mathrm{b}}$ Molecular, Cellular and Developmental Biology and ${ }^{\mathrm{C} B i o l o g i c a l}$ \\ Chemistry, University of Michigan, Ann Arbor, Mich., USA; ${ }^{d}$ INSERM U845, University Paris Descartes, Paris, France
}

\section{Key Words}

Autophagy · Catabolism · Lysosome

\begin{abstract}
With regard to cell biology, one area of focus that has shifted back and forth over the years has been the relative emphasis on catabolic versus anabolic processes: the breakdown of glucose, the synthesis of DNA, the oxidation of pyruvate, the biogenesis of membranes, protein degradation, and protein synthesis. Historically, the majority of studies concerned with degradation dealt with the production of energy; however, the analysis of the ubiquitin-proteasome system revealed the importance of protein degradation for controlling various aspects of cell physiology. The ubiquitin-proteasome system is limited primarily to targeting individual proteins for destruction, but cells also have to deal with larger structures that are damaged, potentially toxic or superfluous, and these substrates, including entire organelles, are the purview of autophagy. As a general definition, autophagy encompasses a range of processes in which the cell degrades parts of itself within the lysosome (or the analogous organelle, the vacuole, in yeast and plants), followed by the release and reuse of the breakdown products. Thus, autophagy is in part a mechanism for cellular recycling, but such a
\end{abstract}

definition belies the importance of the different autophagic processes in cell and organismal function and homeostasis. Indeed, defects in autophagy are associated with many human diseases and metabolic disorders. Here, we provide a brief overview of the mechanism of autophagy and some of the physiological roles in which this process is involved.

Copyright $\odot 2013$ S. Karger AG, Basel

\section{Morphology}

There are several types of autophagy, which differ with regard to their substrates and mechanism of sequestration. For example, chaperone-mediated autophagy is used for the degradation of proteins containing a particular pentapeptide motif, and the targeted substrates must be unfolded for direct translocation across the lysosomal membrane [1]. In contrast, macroautophagy involves the sequestration of parts of the cytoplasm, including large protein complexes and organelles, within a double-membrane cytosolic vesicle that ultimately fuses with the lysosome/vacuole to allow the degradation of its cargo [2]. In this review, we will focus on macroautophagy.

Macroautophagy requires the dynamic rearrangement of significant amounts of subcellular membranes. Con-

\section{KARGER}

E-Mail karger@karger.com

www.karger.com/jin
(C) 2013 S. Karger AG, Basel

1662-811X/13/0055-0427\$38.00/0
Dr. Patrice Codogno

INSERM U845

University Paris Descartes

FR-75015 Paris (France)

E-Mail patrice.codogno@inserm.fr 


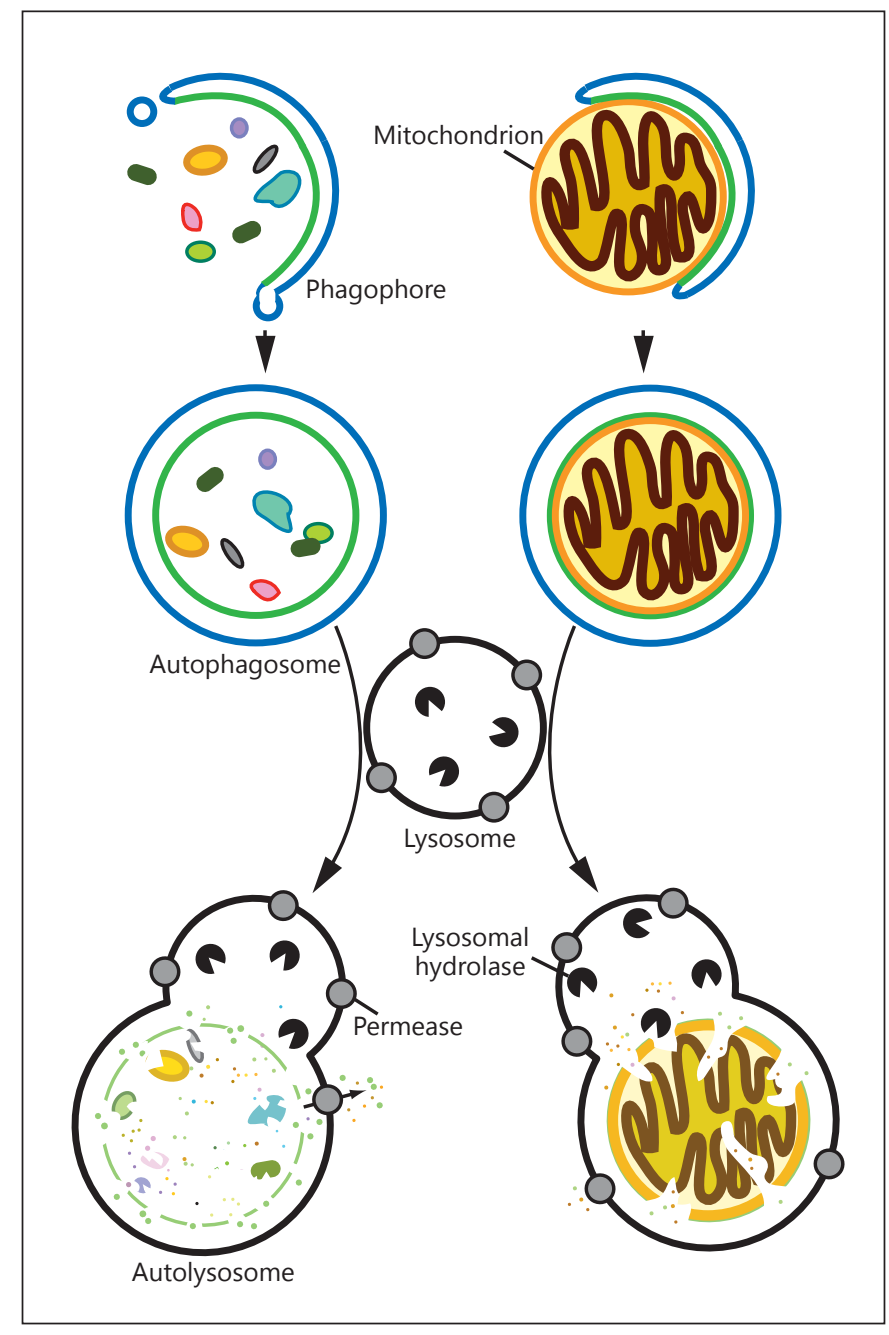

Fig. 1. A schematic representation of macroautophagy in mammalian cells. The initial sequestering structure, the phagophore, expands to sequester cargo in the form of bulk cytoplasm (in nonselective autophagy) or particular targets including intact organelles (in selective types of autophagy such as selective mitochondria degradation and mitophagy, depicted on the right). Upon completion, the cargo is enclosed within a double-membrane autophagosome. Fusion with a lysosome provides access to a wide range of hydrolases that break down the inner autophagosome vesicle along with the cargo. The resulting macromolecules are released back into the cytosol for reuse. A possible intermediate structure, the amphisome, is not depicted. See text for additional details.

sidering that this is essentially the only process for the elimination of damaged organelles, it may be simplest to think about the structural aspects of macroautophagy from this viewpoint. That is, how can a cell sequester an organelle and move it from the cytoplasm into the lyso- some/vacuole lumen? Obviously, an organelle cannot be translocated directly across the limiting membrane of the lysosome/vacuole. For subcellular transport, the cell avoids the repeated problem of membrane translocation in large part through the use of vesicles. That is, many proteins translocate into the endoplasmic reticulum, but then move to subsequent locations inside of transient vesicles in a process that involves membrane fusion rather than additional translocation; this enables even folded proteins, or protein complexes, to move from one membrane-bound compartment to another. So, if a vesicle can be used to avoid the need for translocation across a membrane, the question then becomes the following: how can the cell sequester a large structure such as an organelle within a vesicle?

It is this demand, the need to sequester large portions of the cytoplasm, which drives the unique morphology of macroautophagy [3]. First, the sequestration process needs a high degree of flexibility; different types and sizes of cargo must be accommodated. Hence, in contrast to most vesicular transport processes, macroautophagy does not utilize a single-sized vesicle. Second, unlike most biosynthetic vesicular transport processes, the vesicle does not bud off from a preexisting organelle with the cargo already present in the lumen. Rather, the sequestering vesicle of macroautophagy expands sequentially and engulfs the cargo during the expansion process. The initial sequestering structure is termed 'the phagophore' (fig. 1). The origin and nucleation of the phagophore remain unclear. Ultimately, the phagophore can be viewed as an 'incomplete autophagosome', the latter being the name of the completed double-membrane sequestering vesicle of macroautophagy. Although referred to by name much less frequently, the phagophore is the more interesting and important structure, being the compartment that actively sequesters cargo. Subsequent to nucleation, the phagophore expands, presumably by membrane addition, rather than by direct lipid synthesis.

A specialized type of phagophore, the omegasome, forms from the endoplasmic reticulum (ER) by lateral movement of the ER membrane [4]. More generally, the phagophore appears to expand via fusion of vesicles that may be derived from various membrane donors including the ER [5], the Golgi complex [6], the plasma membrane [7], and possibly the mitochondria [8]. Although the details are not known, the emerging picture is that normal transport machinery that functions during periods of cell growth is 'hijacked' by protein modifications and/or the use of specific protein components to direct membrane flow into the macroautophagic pathway dur- 
ing stress conditions. In yeast, the donor membrane is initially directed to a tubulovesicular cluster [9], which then matures into a phagophore assembly site (PAS). The PAS subsequently develops into a phagophore and then into an autophagosome.

Coat proteins have not been detected associated with the phagophore or autophagosome. Thus, it is not known how the curvature of these structures is determined. Macroautophagy can be nonspecific, sequestering bulk cytoplasm, or it can be highly specific (fig. 1); in the latter case, the phagophore may expand directly around the cargo, in association with ligand molecules on the target and through the interaction of receptors and scaffold proteins. In this case, the cargo may determine the curvature of the sequestering membrane.

After completion, the autophagosome moves to, and fuses with, a lysosome/vacuole. In mammalian cells in particular, the autophagosome may first fuse with an endosome to generate an amphisome, which ultimately fuses with a lysosome. The product of an autophagosome/ amphisome fusing with a lysosome is termed 'an autolysosome'. In fungi and plants, the inner vesicle of the autophagosome is released into the vacuole lumen, where it is termed 'an autophagic body'. Lysis of the autophagosome inner membrane is followed by breakdown of the cargo through the action of lysosomal/vacuolar hydrolases and through release of the degradation products through membrane permeases.

\section{Machinery}

The molecular machinery of macroautophagy was only identified and characterized within the last 15 years, suggesting in part that most of these components function primarily within this pathway, although a few exceptions have been discovered and their numbers continue to increase [10]. Genetic screens in fungi allowed the identification of most of the currently known autophagyrelated (ATG) proteins, and approximately half of the fungal genes encoding these proteins are conserved from yeast to human. Many of the genes that do not have clear homologs in higher eukaryotes are involved in selective types of autophagy; however, selective degradation of organelles clearly occurs in all eukaryotes, suggesting that even in the absence of homologs there will be proteins with analogous functions. An increasing number of ATG proteins that lack fungal homologs are being identified in mammals, which is likely accounted for by their increasing complexity. The ATG proteins can be grouped in dif-

An Overview of Autophagy ferent ways. These include the stage of the process in which they function, the temporal order in which they are recruited to the site of autophagosome formation, or by interacting groups of proteins. Here, we consider them in a slightly different manner, by viewing the process in its entirety.

Macroautophagy occurs at a constitutive basal level, but it is upregulated in response to various types of stress. In addition, the process needs to be kept in check because too much self-degradation can lead to cell death. Accordingly, there are various inputs that regulate the induction of macroautophagy. These include plasma membrane and intracellular sensors that respond to changes in the environment or within the cell, including the presence or absence of amino acids, nitrogen, glucose, growth factors, and reactive oxygen species. Among the ATG proteins, there is a primary group of components that are considered to be involved in an early stage of the signaling process, which is often referred to in yeast as the Atg1 kinase complex. The corresponding proteins include Atg1 (ULK1 and ULK2 in mammals), the accessory factor Atg13 (ATG13) and several regulatory subunits that are less well characterized. The latter consist of a subcomplex of Atg17 (RB1CC1/FIP200)-Atg31-Atg29 and possibly other proteins such as Atg20 and Atg24. In mammals, ATG101 is also part of the complex. Part of the uncertainty regarding the composition of the Atg1 kinase complex is that the holocomplex has not been carefully examined, and the exact function of Atg1 or ULK1/2 is not known; however, these proteins are often considered to be among the initial ones that dictate the site of autophagosome formation.

Generally thought to act downstream of the Atg1 kinase complex is a lipid kinase, Vps34 (PIK3C3 in mammals), which is a class III phosphatidylinositol (PtdIns) 3-kinase. Vps34 is found in two complexes, and complex I is specific to macroautophagy [11]. This complex includes Vps15 (PIK3R4, a presumed regulatory component), Vps30/Atg6 (BECN1) and Atg14 (ATG14). One of the key functions of the PtdIns 3-kinase complex is the generation of PtdIns3P, a phosphoinositide that serves to recruit other factors involved in the autophagosome formation process. For example, Atg18 (mammalian WIPI1 and WIPI2) is a PtdIns3P binding protein, which is involved in the movement of Atg9 (ATG9).

Atg9 is a transmembrane protein that has been the focus of many studies aimed at understanding the membrane movement that plays a critical role in phagophore nucleation and expansion. Some studies suggest that Atg9 transits between the forming autophagosome and periph- 
eral sites (the tubulovesicular clusters), carrying membrane from various donor organelles [12], although the details of such a mechanism are not known. The efficient movement of Atg9 to the site of autophagosome formation (the PAS in yeast) requires Atg23 and Atg27 [13-15]. The return of Atg9, or its release from the PAS, involves Atg2 in a complex with Atg18, as well as Atg1-Atg13.

Perhaps the most studied set of proteins involved in macroautophagy consists of two ubiquitin-like proteins, Atg8 and Atg12, and the components involved in their posttranslational modification [16]. In brief, Atg8 (mammalian members of the MAP1LC3/LC3 or GABARAP subfamilies) is proteolytically processed by the Atg4 (ATG4) protease, activated by Atg7 (ATG7) and then conjugated at its $\mathrm{C}$-terminal glycine through the action of Atg3 (ATG3) to phosphatidylethanolamine. Atg12 (ATG12) is also activated by Atg7 and subsequently conjugated through its C-terminal glycine to an internal lysine residue of Atg5 (ATG5). Atg5 then binds Atg16 (ATG16L1) noncovalently, and the latter dimerizes. The function(s) of the ubiquitin-like protein conjugation systems is not known. Atg8 plays a role in determining the size of the autophagosome [17] and also in recognizing cargo during selective types of macroautophagy. After autophagosome completion, Atg8 is removed from the outer surface of the vesicle by a second Atg4-dependent cleavage in a process termed 'deconjugation'. This event may be involved in triggering the disassembly of the entire Atg protein complex (these proteins do not remain associated with the completed autophagosome) and in preventing premature fusion of the incomplete autophagosome with the vacuole or lysosome.

Although not considered part of the 'core' Atg machinery involved in autophagosome formation [18], there are other proteins that play critical roles in different steps of macroautophagy. For example, in Saccharomyces cerevisiae, Atg19, Atg32, Atg34 and Atg36 are involved in selective types of macroautophagy [19, 20]. Atg19 and Atg34 function as receptors for the biosynthetic delivery of resident vacuolar hydrolases, whereas Atg32 and Atg36 are receptors for the selective degradation of mitochondria and peroxisomes, respectively. All of these processes also utilize Atg11, a scaffold protein. After fusion of the autophagosome with the vacuole/lysosome, the autophagosome inner membrane must be lysed, in a process that requires the Atg15 lipase [21,22], and the cargo degraded by a range of vacuolar hydrolases. Finally, the breakdown products are released back into the cytosol through membrane permeases including Atg22 [23], where they are used as building blocks for anabolic processes, or to gen- erate energy. Although the precise mechanism through which macroautophagy acts selectively in mammalian cells or the nature of the breakdown and efflux processes are not well characterized, they are likely to be similar overall to those in yeast.

\section{Autophagy in Physiology}

Autophagy is induced in cells after many stressful situations such as starvation, hypoxia and infection, among others. Its finely tuned regulation is essential to maintain cell and tissue homeostasis [24]. Stimulation of autophagy during periods of starvation is an evolutionarily conserved response to stress in eukaryotes [2]. Under starvation conditions, the degradation of proteins and lipids allows the cell to adapt its metabolism and meet its energy needs. The stimulation of autophagy plays a major role at birth in maintaining energy levels in various tissues after the maternal nutrient supply via the placenta ceases [25]. Furthermore, pharmacological and genetic downregulation of autophagy induces rapid cell death after starvation in cells [26].

Autophagy is also essential during development and differentiation. The pre-implantation period after oocyte fertilization is dependent on autophagic degradation of components of the oocyte cytoplasm, e.g., elimination of maternal mRNAs [27] and paternal mitochondria [28, 29]. Autophagy is also implicated in the elimination of apoptotic bodies generated during naturally occurring cell death associated with embryonic development [30]. Autophagy remodeling of the cytoplasm is involved in the differentiation of erythrocytes, lymphocytes and adipocytes [31]. Moreover, autophagy is crucial for the homeostasis of immune cells and contributes to the regulation of self-tolerance [32]. Induction of autophagy during caloric restriction may contribute to the observed extension of life span in rats. Recent data have shown that the induction of autophagy increases longevity in a large variety of species [33]. This anti-aging effect likely depends, at least in part, on the quality control function of autophagy, which limits the accumulation of aggregation-prone protein and damaged mitochondria (fig. 1).

\section{Autophagy in Pathology}

As described above, autophagy is essential to eliminate many harmful components in cells such as protein aggregates, damaged organelles and intracellular patho- 
gens. It is thus unsurprising that dysregulation of this process has important consequences and is implicated in many diseases, including Huntington's, Alzheimer's and Parkinson's diseases, which are characterized by the accumulation of protein aggregates in the brain and in other tissues such as muscle [31], as well as in liver fibrosis [34]. In the heart, basal autophagy is necessary to maintain cellular homeostasis and is upregulated in response to stress in hypertensive heart disease, heart failure, cardiac hypertrophy, and ischemia-reperfusion injury [35]. In the pancreas, autophagy is required to maintain the architecture and function of pancreatic $\beta$-cells [36]. Defective hepatic autophagy likely contributes to insulin resistance and to a predisposition to type 2 diabetes and obesity [37]. Given its role in the elimination of intracellular pathogens (bacteria, viruses and parasites), autophagy also contributes to innate immunity [38]. Recently, polymorphisms of the genes that encode ATG16L1 and IRGM, two autophagy proteins essential for the elimination of intracellular pathogens have been associated with Crohn's disease, a chronic inflammatory bowel disease [39].

Amino acids produced by autophagy in the muscles and liver can be used for gluconeogenesis in the liver [40] and can contribute to the production of ATP by entering the tricarboxylic acid cycle. Degradation of liver lipid droplets by autophagy, via lipophagy, contributes to the generation of free fatty acids that are oxidized in the mitochondria [41]. Moreover, hepatocyte-specific Atg7knockout mice exhibit elevated levels of hepatic lipids.

Decreases in hepatic autophagy are observed in both genetic and dietary mouse models of obesity and insulin resistance [37]. This effect has an impact upon ER function, including the response to stress. Restoration of ATG7 expression limits obesity-dependent ER stress and rescues insulin resistance and glucose tolerance. However, autophagy plays an opposite role in white adipose tissue, where its inhibition decreases white adipose mass and enhances insulin sensitivity $[42,43]$. The adiposespecific deletion of Atg7 also favors the oxidation of free fatty acids by increasing the proportion of brown adipocytes, leading to a lean body mass $[42,43]$. The effects of pharmacological manipulation of autophagy in obese patients thus remain uncertain, unless liver autophagy can be specifically targeted [44]. Autophagy is involved not only in the regulation of metabolism in the peripheral tissues, but also in regulating food intake via the brain, although its role in this process remains to be clearly demonstrated $[45,46]$.

An Overview of Autophagy
Whereas cancer is frequently associated with defects in autophagy, the role of autophagy is clearly complex and dependent on cancer stage and context. Autophagy acts as a tumor-suppressing mechanism, but is also required in the later stages of tumor progression to enable tumor cells to cope with metabolic stress [47]. Several of the functions of autophagy, such as the elimination of defective organelles, which reduces oxidative stress and prevents DNA damage, also contribute to its tumor-suppressive effect [48]. Remarkably, autophagy facilitates effectiveglucose uptake and glycolytic fluxin RAS-transformed cells [49]. Moreover, the loss of autophagy in RAS-transformed cells is associated with reduced oxygen consumption and lower levels of the tricarboxylic acid intermediates citrate, aconitate and isocitrate [50]. The high basal level of autophagy observed in tumors with RAS mutations is required for cancer cell survival [51]. In these tumors, autophagy constitutes an Achilles' heel that could prove useful in the fight against cancer. However, inhibiting autophagy is a challenging prospect, as in many tumors, autophagy serves as a stress response to anticancer treatments $[47,52]$.

\section{Future Directions}

Recent years have witnessed advances in our understanding of the origin of the membranes required to form autophagosomes. Whether the origin of the membrane involved in this process varies depending on the stimulus that triggers autophagy remains an unanswered question. A recent proteomics approach has revealed hundreds of interactions between human proteins and the core autophagic machinery, suggesting some hitherto unknown aspects of autophagy regulation that could lead to a better understanding of its integration into cell function [53]. Knowing more about the structure of proteins belonging to the core machinery of autophagy could also accelerate the design of drugs to specifically modulate the process [54]. The precise regulation of autophagy is an exciting challenge for therapeutic applications in some major human diseases [55].

References

Arias E, Cuervo AM: Chaperone-mediated autophagy in protein quality control. Curr Opin Cell Biol 2011;23:184-189.

2 Yang Z, Klionsky DJ: Mammalian autophagy: core molecular machinery and signaling regulation. Curr Opin Cell Biol 2010;22:124131. 
-3 Eskelinen E-L, Reggiori F, Baba M, Kovacs $\mathrm{AL}$, Seglen PO: Seeing is believing: the impact of electron microscopy on autophagy research. Autophagy 2011;7:935-956.

-4 Axe EL, Walker SA, Manifava M, Chandra P, Roderick HL, Habermann A, Griffiths G, Ktistakis NT: Autophagosome formation from membrane compartments enriched in phosphatidylinositol 3-phosphate and dynamically connected to the endoplasmic reticulum. J Cell Biol 2008;182:685-701.

5 Lynch-Day MA, Bhandari D, Menon S, Huang J, Cai H, Bartholomew CR, Brumell JH, Ferro-Novick S, Klionsky DJ: Trs85 directs a Ypt1 GEF, TRAPPIII, to the phagophore to promote autophagy. Proc Natl Acad Sci USA 2010;107:7811-7816.

6 Yen W-L, Shintani T, Nair U, Cao Y, Richardson BC, Li Z, Hughson FM, Baba M, Klionsky DJ: The conserved oligomeric Golgi complex is involved in double-membrane vesicle formation during autophagy. J Cell Biol 2010; 188:101-114.

7 Ravikumar B, Moreau K, Jahreiss L, Puri C, Rubinsztein DC: Plasma membrane contributes to the formation of pre-autophagosomal structures. Nat Cell Biol 2010;12:747-757.

-8 Hailey DW, Rambold AS, Satpute-Krishnan P, Mitra K, Sougrat R, Kim PK, LippincottSchwartz J: Mitochondria supply membranes for autophagosome biogenesis during starvation. Cell 2010;141:656-667.

-9 Mari M, Griffith J, Rieter E, Krishnappa L, Klionsky DJ, Reggiori F: An Atg9-containing compartment that functions in the early steps of autophagosome biogenesis. J Cell Biol 2010;190:1005-1022.

10 Codogno P, Mehrpour M, Proikas-Cezanne T: Canonical and non-canonical autophagy: variations on a common theme of self-eating? Nat Rev Mol Cell Biol 2011;13:7-12.

-11 Kihara A, Noda T, Ishihara N, Ohsumi Y: Two distinct Vps34 phosphatidylinositol 3-kinase complexes function in autophagy and carboxypeptidase Y sorting in Saccharomyces cerevisiae. J Cell Biol 2001;152:519530.

-12 Reggiori F, Tucker KA, Stromhaug PE, Klionsky DJ: The Atg1-Atg13 complex regulates Atg9 and Atg23 retrieval transport from the pre-autophagosomal structure. Dev Cell 2004;6:79-90.

13 Tucker KA, Reggiori F, Dunn WA Jr, Klionsky DJ: Atg23 is essential for the cytoplasm to vacuole targeting pathway and efficient autophagy but not pexophagy. J Biol Chem 2003;278:48445-48452.

14 Legakis JE, Yen W-L, Klionsky DJ: A cycling protein complex required for selective autophagy. Autophagy 2007;3:422-432.

15 Yen W-L, Legakis JE, Nair U, Klionsky DJ: Atg27 is required for autophagy-dependent cycling of Atg9. Mol Biol Cell 2007; 18:581593.

16 Geng J, Klionsky DJ: The Atg8 and Atg12 ubiquitin-like conjugation systems in macroautophagy. EMBO Rep 2008;9:859-864.
17 Xie Z, Nair U, Klionsky DJ: Atg8 controls phagophore expansion during autophagosome formation. Mol Biol Cell 2008;19:32903298.

18 Xie Z, Klionsky DJ: Autophagosome formation: core machinery and adaptations. Nat Cell Biol 2007;9:1102-1109.

19 Mijaljica D: Receptor protein complexes are in control of autophagy. Autophagy 2012;8: 1701-1705.

20 Motley AM, Nuttall JM, Hettema EH: Pex3anchored Atg36 tags peroxisomes for degradation in Saccharomyces cerevisiae. EMBO J 2012;31:2852-2868.

21 Epple UD, Suriapranata I, Eskelinen E-L, Thumm M: Aut5/Cvt17p, a putative lipase essential for disintegration of autophagic bodies inside the vacuole. J Bacteriol 2001;183:59425955.

22 Teter SA, Eggerton KP, Scott SV, Kim J, Fischer AM, Klionsky DJ: Degradation of lipid vesicles in the yeast vacuole requires function of Cvt17, a putative lipase. J Biol Chem 2001;276:2083-2087.

23 Yang Z, Huang J, Geng J, Nair U, Klionsky DJ: Atg22 recycles amino acids to link the degradative and recycling functions of autophagy. Mol Biol Cell 2006;17:5094-5104.

24 Kroemer G, Marino G, Levine B: Autophagy and the integrated stress response. Mol Cell 2010;40:280-293.

25 Kuma A, Hatano M, Matsui M, Yamamoto A Nakaya H, Yoshimori T, Ohsumi Y, Tokuhisa $\mathrm{T}$, Mizushima N: The role of autophagy during the early neonatal starvation period. Nature 2004;432:1032-1036.

26 Boya P, Gonzalez-Polo RA, Casares N, Perfettini JL, Dessen P, Larochette N, Metivier D, Meley D, Souquere S, Yoshimori T, Pierron G, Codogno P, Kroemer G: Inhibition of macroautophagy triggers apoptosis. Mol Cell Biol 2005;25:1025-1040.

27 Tsukamoto S, Kuma A, Murakami M, Kishi C, Yamamoto A, Mizushima N: Autophagy is essential for preimplantation development of mouse embryos. Science 2008;321:117-120.

28 Sato M, Sato K: Degradation of paternal mitochondria by fertilization-triggered autophagy in C. elegans embryos. Science 2011;334: 1141-1144.

29 Al Rawi S, Louvet-Vallee S, Djeddi A, Sachse M, Culetto E, Hajjar C, Boyd L, Legouis R, Galy V: Postfertilization autophagy of sperm organelles prevents paternal mitochondrial DNA transmission. Science 2011;334:11441147

$30 \mathrm{Qu}$ X, Zou Z, Sun Q, Luby-Phelps K, Cheng P, Hogan RN, Gilpin C, Levine B: Autophagy gene-dependent clearance of apoptotic cells during embryonic development. Cell 2007; 128:931-946.
31 Ravikumar B, Sarkar S, Davies JE, Futter M, Garcia-Arencibia M, Green-Thompson ZW, Jimenez-Sanchez M, Korolchuk VI, Lichtenberg M, Luo S, Massey DC, Menzies FM, Moreau K, Narayanan U, Renna M, Siddiqi FH, Underwood BR, Winslow AR, Rubinsztein DC: Regulation of mammalian autophagy in physiology and pathophysiology. Physiol Rev 2010;90:1383-1435.

-32 Nedjic J, Aichinger M, Mizushima N, Klein L: Macroautophagy, endogenous MHC II loading and $\mathrm{T}$ cell selection: the benefits of breaking the rules. Curr Opin Immunol 2009;21: 92-97.

33 Rubinsztein DC, Marino G, Kroemer G: Autophagy and aging. Cell 2011;146:682-695.

34 Hidvegi T, Ewing M, Hale P, Dippold C, Beckett C, Kemp C, Maurice N, Mukherjee A, Goldbach C, Watkins S, Michalopoulos G, Perlmutter DH: An autophagy-enhancing drug promotes degradation of mutant $\alpha_{1-}$ antitrypsin $\mathrm{Z}$ and reduces hepatic fibrosis. Science 2010;329:229-232

- 35 Nakai A, Yamaguchi O, Takeda T, Higuchi Y, Hikoso S, Taniike M, Omiya S, Mizote I, Matsumura $\mathrm{Y}$, Asahi M, Nishida K, Hori M, Mizushima N, Otsu K: The role of autophagy in cardiomyocytes in the basal state and in response to hemodynamic stress. Nat Med 2007;13:619-624.

-36 Ebato C, Uchida T, Arakawa M, Komatsu M, Ueno T, Komiya K, Azuma K, Hirose T, Tanaka K, Kominami E, Kawamori R, Fujitani Y, Watada H: Autophagy is important in islet homeostasis and compensatory increase of beta cell mass in response to high-fat diet. Cell Metab 2008;8:325-332.

37 Yang L, Li P, Fu S, Calay ES, Hotamisligil GS: Defective hepatic autophagy in obesity promotes ER stress and causes insulin resistance. Cell Metab 2010;11:467-478.

38 Deretic V: Autophagy as an innate immunity paradigm: expanding the scope and repertoire of pattern recognition receptors. Curr Opin Immunol 2012;24:21-31.

39 Virgin HW, Levine B: Autophagy genes in immunity. Nat Immunol 2009;10:461-470.

40 Rabinowitz JD, White E: Autophagy and metabolism. Science 2010;330:1344-1348.

-41 Singh R, Kaushik S, Wang Y, Xiang Y, Novak I, Komatsu M, Tanaka K, Cuervo AM, Czaja MJ: Autophagy regulates lipid metabolism. Nature 2009;458:1131-1135.

42 Zhang Y, Goldman S, Baerga R, Zhao Y, Komatsu M, Jin S: Adipose-specific deletion of autophagy-related gene 7 (Atg7) in mice reveals a role in adipogenesis. Proc Natl Acad Sci USA 2009;106:19860-19865.

43 Singh R, Xiang Y, Wang Y, Baikati K, Cuervo AM, Luu YK, Tang Y, Pessin JE, Schwartz GJ, Czaja MJ: Autophagy regulates adipose mass and differentiation in mice. J Clin Invest 2009; 119:3329-3339.

44 Codogno P, Meijer AJ: Autophagy: a potential link between obesity and insulin resistance. Cell Metab 2010;11:449-451. 
45 Kaushik S, Rodriguez-Navarro JA, Arias E, Kiffin R, Sahu S, Schwartz GJ, Cuervo AM, Singh R: Autophagy in hypothalamic AgRP neurons regulates food intake and energy balance. Cell Metab 2011;14:173-183.

46 Meng Q, Cai D: Defective hypothalamic autophagy directs the central pathogenesis of obesity via the I $\mathrm{B}$ kinase $\beta(\mathrm{IKK} \beta) / \mathrm{NF}-\kappa \mathrm{B}$ pathway. J Biol Chem 2011;286:32324-32332.

47 Cheong $\mathrm{H}, \mathrm{Lu} \mathrm{C}$, Lindsten T, Thompson CB: Therapeutic targets in cancer cell metabolism and autophagy. Nat Biotechnol 2012;30:671678.

48 White E: Deconvoluting the context-dependent role for autophagy in cancer. Nat Rev Cancer 2012;12:401-410.
9 Lock R, Roy S, Kenific CM, Su JS, Salas E, Ronen SM, Debnath J: Autophagy facilitates glycolysis during Ras-mediated oncogenic transformation. Mol Biol Cell 2011;22:165178.

50 Guo JY, Chen HY, Mathew R, Fan J, Strohecker AM, Karsli-Uzunbas G, Kamphorst JJ, Chen G, Lemons JM, Karantza V, Coller HA, Dipaola RS, Gelinas C, Rabinowitz JD, White E: Activated Ras requires autophagy to maintain oxidative metabolism and tumorigenesis. Genes Dev 2011;25:460-470.

51 Yang S, Wang X, Contino G, Liesa M, Sahin E, Ying $\mathrm{H}$, Bause A, Li Y, Stommel JM, Dell'Antonio G, Mautner J, Tonon G, Haigis M, Shirihai OS, Doglioni C, Bardeesy N, Kimmelman AC: Pancreatic cancers require autophagy for tumor growth. Genes Dev 2011; 25:717-729.
2 Janku F, McConkey DJ, Hong DS, Kurzrock R: Autophagy as a target for anticancer therapy. Nat Rev Clin Oncol 2011;8:528-539.

53 Behrends C, Sowa ME, Gygi SP, Harper JW: Network organization of the human autophagy system. Nature 2010;466:68-76.

54 Miller S, Tavshanjian B, Oleksy A, Perisic O, Houseman BT, Shokat KM, Williams RL: Shaping development of autophagy inhibitors with the structure of the lipid kinase Vps34. Science 2010;327:1638-1642.

55 Rubinsztein D, Codogno P, Levine B: Autophagy modulation as a potential therapeutic target for diverse diseases. Nat Rev Drug Discov 2012;11:709-730. 